\title{
Fish Fin Aspiration: An Unusual Type of Lower Airway Foreign Body in a Chinese Adult
}

\author{
Chun-Yao Lin MD, Shiu-Feng Huang MD PhD, Chou-Chin Lan MD PhD, Yao-Kuang Wu MD, \\ Chun-Yao Huang MD, Yi-Chih Huang MD, Chor-Shen Lim MD, and Mei-Chen Yang MD
}

\begin{abstract}
Foreign body aspiration into the lower airway is rare in adults, and typically occurs in individuals of advanced age or with underlying neurological or medical conditions. The most common type of lower airway foreign body is organic substances, in particular chicken or fish bones. In many patients a history of an acute choking event is not found, and symptoms are non-specific and may be attributed to other medical conditions. Herein we report the unique case of an 80 -year-old Chinese woman who aspirated a fish fin that was undiagnosed for 3 years. Key words: fish fin; lower airway foreign body; aspiration; bronchoscopy. [Respir Care 2013;58(3):e20-e22. () 2013 Daedalus Enterprises]
\end{abstract}

\section{Introduction}

Foreign body aspiration into the lower airway is rare in adults, and typically occurs when there is a predisposing factor such as older age, the use of sedative or hypnotic medications, or medical conditions. ${ }^{1,2}$ The most common type of materials aspirated into the lower airway are organic substances, in particular chicken or fish bones. ${ }^{1,2}$ The fish fin is generally thought to be inedible; however, Chinese have a fondness for eating fish fin because of the tasty flesh surrounding the fin. ${ }^{3}$ Fish fin improperly swallowed into the gastrointestinal tract has been reported ${ }^{4}$; however, to the best of our knowledge fish fin aspiration into the lower airway has never been reported. Herein we

Dr Lin is affiliated with the Division of Pulmonary, Critical Care, and Sleep Medicine, Chang Gung Memorial Hospital, Keelung City, Taiwan. Dr Shiu-Feng Huang is affiliated with the Department of Clinical $\mathrm{Pa}$ thology; and Drs Lan, Wu, Chun-Yao Huang, Yi-Chih Huang, Lim, and Yang are affiliated with the Division of Pulmonary and Critical Care Medicine, Buddhist Tzu Chi General, Hospital, New Taipei City, Taiwan. Drs Shiu-Feng Huang, Lan, Wu, and Yang are also affiliated with the School of Medicine, Tzu-Chi University, Hualien, Taiwan.

The authors have disclosed no conflicts of interest.

Correspondence: Mei-Chen Yang MD, Division of Pulmonary and Critical Care Medicine, Department of Internal Medicine, Buddhist Tzu Chi General Hospital, Taipei Branch, 289 Jianguo Road, Xindian District, New Taipei City 23142, Taiwan. E-mail: mimi3461@ms45.hinet.net.

DOI: $10.4187 /$ respcare. 01971 report a case of fish fin aspiration that was undiagnosed for 3 years. This report was approved by the ethics committee and institutional review board of Buddhist Tzu Chi General Hospital, Taipei branch.

\section{Case Report}

An 80-year-old Chinese woman with history of senile dementia was seen with a 1 month history of progressive shortness of breath, chest tightness, and body weight loss. Her history was notable for a 3 year history of persistent cough, for which she had been treated symptomatically. One year prior to being seen by the author, she was treated with levofloxacin for bilateral pneumonia, but the chronic cough persisted. Three months prior to being seen, she was again treated with levofloxacin for fever and productive cough. Chest radiograph at that time showed minimal right lower lung infiltration.

Physical examination revealed the patient to be afebrile, with a breathing frequency of 28 breaths/min, heart rate of 96 beats/min, and a blood pressure of $126 / 68 \mathrm{~mm} \mathrm{Hg}$. She appeared dyspneic, crackles were noted over the right lower lung, and foul smelling sputum was noted when she coughed. Her caregiver denied any history of choking or foreign body aspiration.

Chest radiograph showed right middle lobe and right lower lobe atelectasis (Fig. 1), and computed tomography (CT) revealed a right hilar soft tissue density with right lower lobe bronchus narrowing, right middle lobe collapse, and right lower lobe localized bronchiectasis (Fig. 2). 


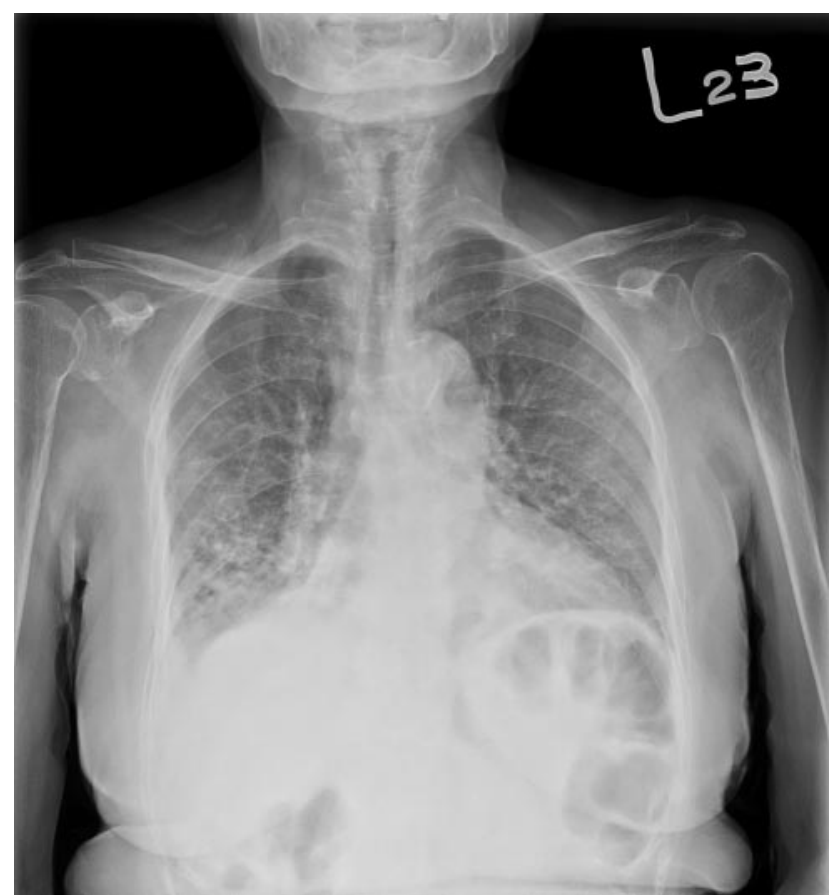

Fig. 1. Chest radiograph reveals right middle and lower lobe atelectasis.

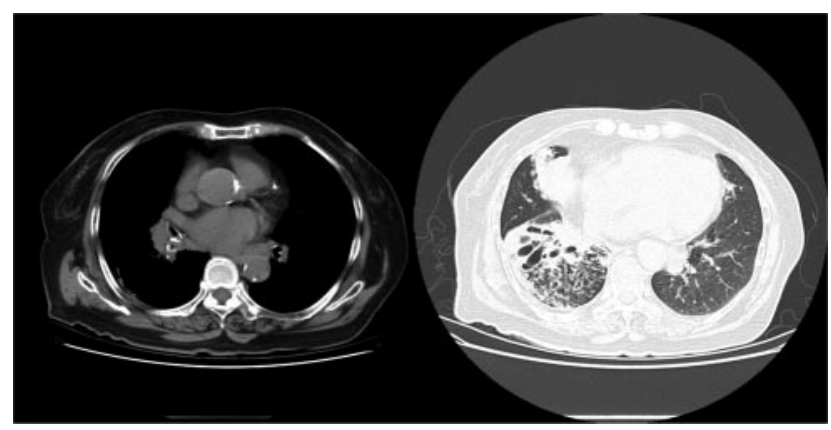

Fig. 2. Computed tomography shows a right hilar soft tissue density, right lower lobe bronchus narrowing, right middle lobe collapse, and right lower lobe inflammation, with bronchiectatic changes.

Augmentin $625 \mathrm{mg}$ BID was given for 1 week, and then bronchoscopy was performed, which revealed a whitish, fragile, long and flat shaped foreign body lodged in the right intermediate bronchus. The proximal end of the foreign body was floating in the lumen of the right intermediate bronchus, and the distal end had a thorn-like portion piercing both the right middle lobe and right lower lobe bronchi (Fig. 3). The mucosa surrounding the foreign body was severely inflamed and hypertrophy was noted. The material was easily cut with a biopsy forceps and removed in several pieces. After removal, a bronchial mucosal biopsy was performed to rule out malignancy.

Histopathology examination of the foreign body revealed dense hyalinized acellular tissue with palisading collagen

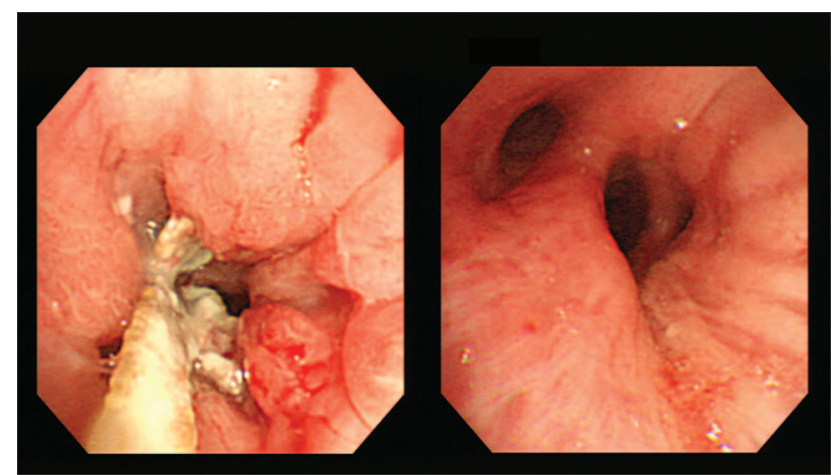

Fig. 3. Left: Initial bronchoscopy shows a fragile, long flat-shaped foreign body with thorn-like projections piercing into the mucosa of right middle and lower bronchial mucosa. Right: One month after removal of the foreign body the bronchial mucosa appears normal.

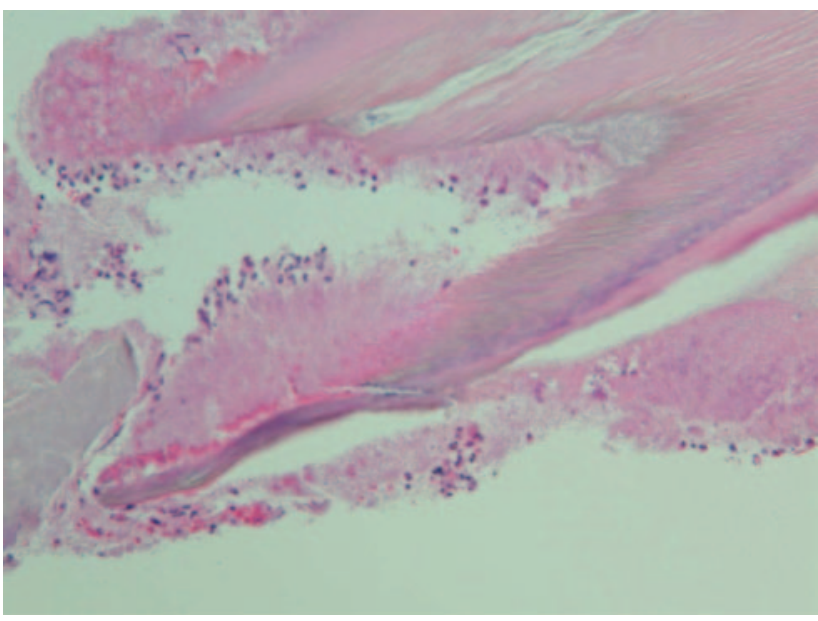

Fig. 4. Histopathological examination of the foreign body revealed dense hyalinized acellular tissue with palisading collagen fibers, compatible with a fish fin. Evidence of acute suppurative inflammation was also noted (hematoxylin and eosin stain, 100×).

fibers, compatible with a fish fin (Fig. 4). No osteocytes were observed. Examination of the biopsy specimen revealed no evidence of malignancy. One month after removal of the foreign body, the patient's cough had completely resolved, and follow-up bronchoscopy revealed complete recovery of the bronchial mucosa (see Fig. 3).

\section{Discussion}

In adults the prevalence of foreign body aspiration into the lower airway increases with age, beginning in the sixth decade of life, and is usually associated with an underlying impairment such as altered mental status or neurological dysfunction. ${ }^{2}$ While many different types of foreign body have been reported, the majority are organic substances (ie, food matter). ${ }^{1,2}$ The nature of aspirated foreign bodies 
in the lower airway varies with different countries, due to eating habits, and has been found to be different in Chinese, as compared to Western, adults. ${ }^{3}$

History, clinical manifestations, and physical signs of lower airway foreign body aspiration are typically variable and non-specific, and will depend on the site where the material is lodged. ${ }^{1,2}$ In older adults, symptoms are often attributed to medical conditions, especially when no clear history of aspiration is apparent, and can be longstanding. ${ }^{5}$ When wedged distally, a foreign body in the lower airway can be innocuous. ${ }^{1}$ In most cases of adult aspiration there is a sudden onset of coughing and choking; however, if the symptoms resolve, most patients do not remember the incident. Reports have indicated that only $25-38 \%$ of adult patients who are found to have a lower airway foreign body remember a choking event.6,7

Chest radiography is the primary imaging modality used to identify a foreign body in the lower airway; however, the false-negative rate of radiographs in adults with suspected aspiration has been reported to be up to $80 \% .{ }^{8}$ This is due to the physical nature of the material aspirated; most foreign material aspirated in adults is organic and therefore radiolucent. Interestingly, the radiopacity of fish bones, which are frequently aspirated, depends on the species of fish. ${ }^{9} \mathrm{CT}$ is more sensitive than radiography for the diagnosis of a lower airway foreign body, but can lack specificity, and identification of a foreign body depends on the slice thickness of the CT images relative to the size of the foreign body. ${ }^{8}$ A negative CT does not exclude the possibility of a foreign body.

Flexible fiberoptic bronchoscopy is the definitive method for the diagnosis and removal of foreign bodies, and is successful in $>90 \%$ of cases. ${ }^{1}$ However, a foreign body is often surrounded by friable granulation tissue, and extraction can be difficult. ${ }^{1}$ When the foreign body is visualized, the entire shape and structure in relationship to the sur- rounding areas should be carefully evaluated before extraction is attempted. ${ }^{1}$

In conclusion, we have reported an unusual case of fish fin aspiration into the lower airway. Different cultural habits can result in a variation of materials that are commonly aspirated. Initial diagnosis of a foreign body in the lower airway can be difficult due to nonspecific signs and symptoms once an initial choking event is forgotten, and chest radiography and CT have high false-negative rates. Fiberoptic bronchoscopy is successful for diagnosis and removal in over $90 \%$ of cases.

\section{REFERENCES}

1. Ramos MB, Fernández-Villar A, Rivo JE, Leiro V, García-Fontán E, Botana MI, et al. Extraction of airway foreign bodies in adults: experience from 1987-2008. Interact Cardiovasc Thorac Surg 2009; 9(3):402-405.

2. Boyd M, Chatterjee A, Chiles C, Chin R Jr. Tracheobronchial foreign body aspiration in adults. South Med J 2009;102(2):171-174.

3. Chen CH, Lai CL, Tsai TT, Lee YC, Perng RP. Foreign body aspiration into the lower airway in Chinese adults. Chest 1997;112(1): 129-133.

4. Bhatia R, Deane AJ, Landham P, Schulte KM. An unusual case of bowel perforation due to fish fin ingestion. Int J Clin Pract 2006; 60(2):229-231.

5. Wu TH, Cheng YL, Tzao C, Chang H, Hsieh CM, Lee SC. Longstanding tracheobronchial foreign body in an adult. Respir Care 2012; 57(5):808-810.

6. Mise K, Jurcev Savicevic A, Pavlov N, Jankovic S. Removal of tracheobronchial foreign bodies in adults using flexible bronchoscopy: experience 1995-2006. Surg Endosc 2009;23(6):1360-1364.

7. Donado Uña JR, de Miguel Poch E, Casado López ME, Alfaro Abreu JJ. Fiber optic bronchoscopy in extraction of tracheo-bronchial foreign bodies in adults. Arch Bronconeumol 1998;34(2): 76-81.

8. Zissin R, Shapiro-Feinberg M, Rozenman J, Apter S, Smorjik J, Hertz M. CT findings of the chest in adults with aspirated foreign bodies. Eur Radiol 2001;11(4):606-611.

9. Ell SR, Sprigg A. The radio-opacity of fishbones-species variation. Clin Radiol 1991;44(2):104-107. 